\title{
El problema genético de los fondos de fiordo Norpatagónicos
}

\author{
José F. Araya-Vergara \\ Departamento de Geografía de la Universidad de Chile, Cas. 3387, Santiago.
}

\begin{abstract}
RESUMEN. Se intenta fundamentar una discusión sobre la génesis de los fondos de fiordo de Norpatagonia. Está demostrado que un piedmont glacial cubrió la región en la Última Glaciación, pero hasta ahora se desconocía sus efectos submarinos en fiordos. Los registros de ecosonda de alta resolución $(3.5 \mathrm{k} \mathrm{Hz})$ en perfiles longitudinales indican dos categorías de cuencas: con explanada de represamiento uniforme y con explanada de represamiento y acumulaciones caóticas. Las primeras muestran dos fases evolutivas: en la inferior, las capas están deformadas y el origen de sus materiales es conjetural; en la superior, las capas laminadas están casi intactas y llenan depresiones sobre la fase inferior; sus materiales son de origen coluvial. Las segundas indican sincronismo genético entre acumulaciones caóticas y capas de la explanada de represamiento.

Consecuentemente, para explanadas de represamiento uniforme, se desconoce aún la génesis de la fase inferior; en cambio, una buena parte de la fase superior puede ser reconstituida: la datación de sus capas superficiales indica edad holocena. Para cuencas con depósitos caóticos, se puede aplicar modelos sobre origen de morrenas submarinas, que se propone llamar polifásicas.
\end{abstract}

\section{The genetical problem of the North Patagonic fjord bottoms}

\begin{abstract}
It is an attempt of founded discussion on the genesis of the fjord bottoms of Northern Patagonia. Even though the operation of a Pleistocene glacial piedmont in this region has been demonstrated, its submarine effect on the fjord basins was until now ignored. The longitudinal high resolution $3.5 \mathrm{k} \mathrm{Hz}$ echo-sounder records, show two types of fjord basins: uniform ponding esplanade and ponding esplanade alternating with chaotic accumulations. In the first, two evolutive phases are indentified; the beds of the lower phase are remolded and the source of its material is conjectural; in exchange, the laminated beds of the upper phase are intact and fill depressions on materials of the lower phase, with sediments supplied by slopes. In the second, there is synchronical construction between chaotic accumulations and the beds of ponding esplanade.

Consequently, for the basins with uniform esplanade, the genesis of the lower phase is ignored yet; in exchange, a good part of the upper phase can be reconstructed; the dating of its superficial beds indicates Holocene age. For basins with chaotic accumulations, models for construction of submarine moraines can be applied. The concept of polyphasic moraines is proposed.
\end{abstract}

\section{INTRODUCCIÓN}

La región de fiordos de Norpatagonia (Fig. 1) ha sido estudiada principalmente desde el punto de vista de la geocronología de las formas subaéreas resultantes de la operación de la deriva glacial en la Última Glaciación y el Holoceno (Heusser y Flint, 1977; Heusser, 1990). La discusión de Heusser (1990) incluye reflexiones sobre los probables ambientes glaciomarinos o glaciolacustres y el modo general de operación del hielo, pero basadas solamente en una aproximación batimétrica de las formas submarinas del piedmont (dominio externo en Fig.1) y asumiendo un comportamiento glacioeustático conjetural para una región englaciada. Las primeras observaciones de reflectores acústicos en el fondo de las cuencas de fiordos típicos (Araya-Vergara, 1996), indican siempre la presencia de fondos planos, estructurados por multicapas laminadas y horizontales, de 3 a 4 m de espesor cada una, sumando po- 


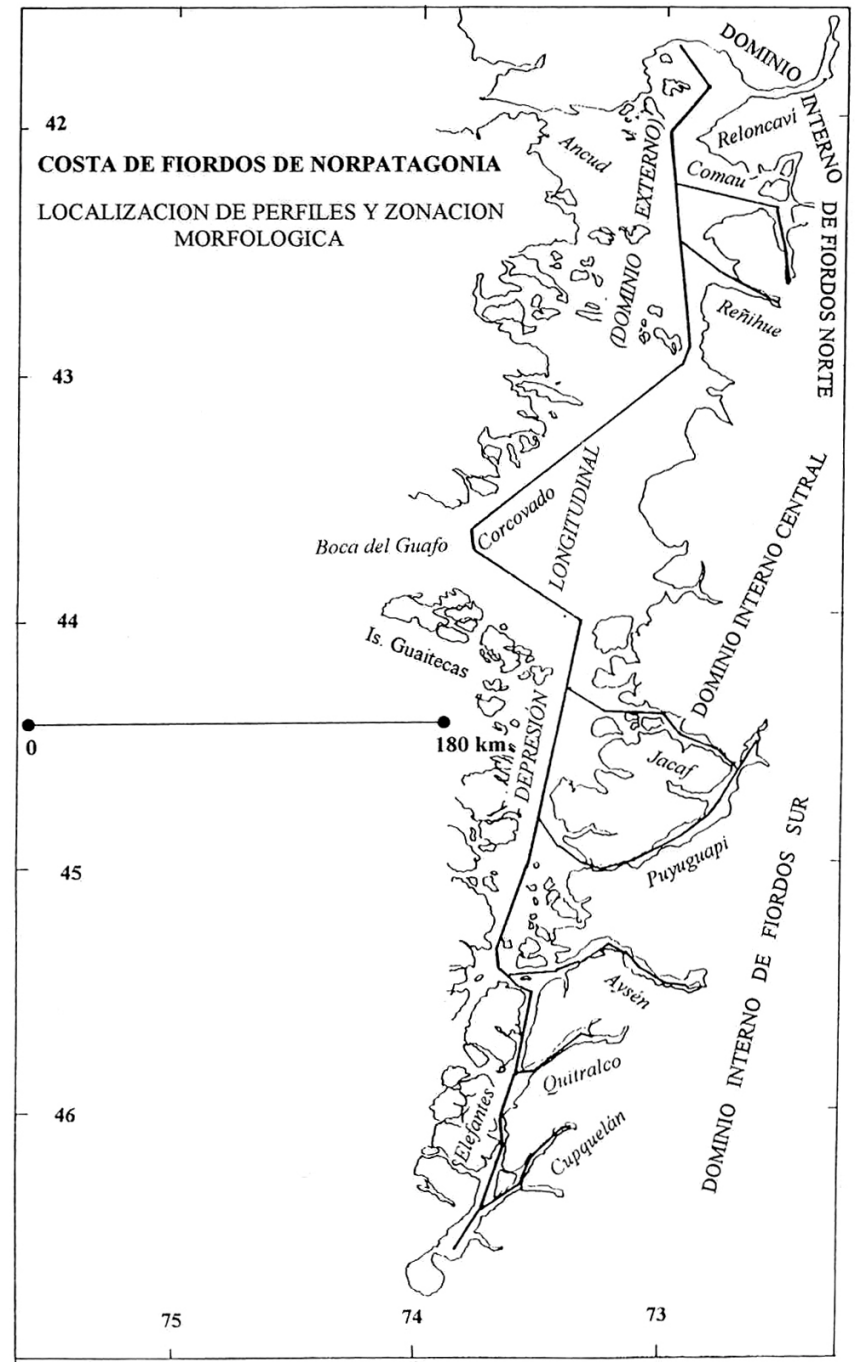

Fig. 1. Las cuencas de fiordo típicas, con explanadas de represamiento uniformes, están ubicadas en el dominio interno. Nótese el emplazamiento especial de Canal Jacaf, el único con formas glacigénicas.

Fig. 1. The typical fjord basins, with uniform ponding esplanades, are located in the inner dominion. See the location of Canal Jacaf, the unique with glacial landforms.

Tabla 1: Clases de reflectores.

Table 1: Classes of reflectors.

\begin{tabular}{|c|c|c|}
\hline Clase & $\begin{array}{l}\text { Espesor de trazo vs. } \\
\text { distancia mínima de } \\
\text { resolución }\end{array}$ & Grado de nitidez \\
\hline Fuerte & Superior & Nítido, tenue, muy tenue \\
\hline Medio & Aproximadamente igual & Idem. \\
\hline Débil & Inferior & idem. \\
\hline
\end{tabular}

tencias de decenas de metros. Para el caso atípico de Canal Jacaf, los fondos planos se alternan con acumulaciones caóticas. En ambos casos, la discusión genética quedó pendiente.

Por lo tanto, el propósito de este trabajo es fundamentar una discusión acerca de la génesis de los fondos de fiordo norpatagónicos.

\section{MATERIALES Y MÉTODOS}

Se usó registros de un ecosonda E. Western de alta resolución (frecuencia $3.5 \mathrm{k} \mathrm{Hz}$ ), correspondientes a perfiles longitudinales a los fiordos, obtenidos en el crucero Cimar-Fiordo 1, realizado en el AGOR Vidal Gormaz (Fig. 1). Debido al cambio en el rango local de profundidades, el registro original de cada transecto se obtuvo a una de las siguientes escalas verticales aproximadas: 1:3.000, 1:1.500 ó 1:750. Al mismo tiempo, las escalas horizontales aproximadas variaron entre 1:45.000 y 1:20.000, para velocidades de crucero de 12 a 6 nudos respectivamente. Así, el factor de exageración vertical de la escala fluctúa entre 10 y 50. Para compensar la posible influencia de los cambios de escala en rasgos como la longitud de pulso, se procedió a correlacionar reflectores guías entre diferentes escalas. A la escala vertical más usada, 1:1.500, la resolución que permite distinguir dos reflectores débiles contiguos es de 1.5 a $3 \mathrm{~m}$ en sentido vertical (1 a $2 \mathrm{~mm}$ en el registro); para reflectores fuertes, difícilmente baja de $5 \mathrm{~m}$ ( 3 a $4 \mathrm{~mm}$ en el registro). Por lo tanto, la base para definir la clase de reflector -en la operación de su seguimiento- fue su espesor relativo en el registro, en función de la distancia mínima de resolución entre dos reflectores delgados (Tabla 1). Para el propósito de este trabajo, es más importante el seguimiento de estructuras de reflectores que el de reflectores individuales.

Para resaltar detalles en las estruc- 
turas de reflectores, fotografías de registro fueron digitalizadas por scanner y procesadas computacionalmente con el programa Corel Photo Paint, aplicando ajustes de contraste, brillo, corrección Gamma y selección de escenas ampliadas.

Como complementación genética, la pendiente fue establecida a partir de la fórmula de la tangente para cada categoría de forma y expresada tanto en ángulo como en altán (log tan del ángulo), ya que el significado de las diferencias de pendiente por unidad angular crece de manera aproximadamente logarítmica, a medida que el ángulo decrece. Para las determinaciones se confeccionó una escala gráfica de pendientes, que contempla la exageración vertical, el factor de distancia horizontal y los rangos de pendiente en grados usando la razón 2, lo que da rangos de clase de alrededor de 3 altanes cada uno. Conjuntamente, para interpretar los reflectores en términos de la naturaleza de los materiales, se consideró que el sonar de $3.5 \mathrm{k} \mathrm{Hz}$ posee una variabilidad de penetración notable: decenas de metros en sedimentos fangosos y blandos y unos pocos metros en algunos substratos arenosos (Carlson, 1989).

Así, la observación de morfoestructuras a partir de reflectores fue dirigida a los siguientes tipos de emplazamiento: umbrales que separan cuencas, vertientes medias a bajas de umbral y explanadas de fondo de cuenca. La elección y observación de expresiones acústicas de formas deposicionales reposó en los elementos básicos de la teoría construida hasta ahora: a) Para umbrales y vertientes: características de reflectores incoherentes de umbral, de vertiente de umbral y de explanada de fondo y su engranamiento con los sedimentos de esta última (Josenhans y Fader, 1989); sistemática de los deslizamientos de terreno en vertientes (Barnes y Lewis, 1991); modelado de facies de turbidez (Porebski et al., 1991); múltiples fuentes y zonación de depósitos y formas (Trincardi et al., 1995); zonación de depósitos de flujos de gravedad en aguas someras (Myrow y Hiscott, 1991) y clasificación de movimientos en masa del litoral afuera (Mulder y Cochonat, 1996). b) Para explanadas de fondo de cuenca: estructura de conjunto entre éstas y las vertientes (The Grape Team, 1990); balance entre deposición y erosión en el espesor de capas de turbiditas (Rothman et al., 1994); importancia de la noción de capacidad en el control de las turbiditas (Hiscott, 1994) y los descubrimientos sobre génesis de lagos subglaciales (Siegert et al., 1996). c) Acerca de la puesta en lugar de depósitos representados por reflectores incoherentes: fenómenos derivados de la puesta en flotación del hielo (Josenhans y Fader, 1989), de calving glaciers y deriva de icebergs (Frezzotti, 1997; Harry et al., 1990), de ruptura de calota (Vaughan, 1993) y de fluctuaciones en el Pleistoceno y en el Holoceno (Barret y Hambrey, 1992; Hansom y Flint, 1989).

\section{RESULTADOS}

En Norpatagonia hay dos tipos de cuencas de fiordo: con explanadas de represamiento uniformes y con explanadas de represamiento alternadas con acumulaciones caóticas. Las Tablas 2 y 3 señalan sus diferencias.

Tabla 2. Pendientes de vertientes I: Fiordos con explanadas de represamiento uniformes.

Table 2. Slope angles: Fjords with uniform ponding esplanade. Symbols as in figures 2 - 9.

\begin{tabular}{|c|c|c|c|c|c|c|c|c|}
\hline \multirow[t]{2}{*}{ Fiordo } & \multicolumn{2}{|c|}{ Umbral rocoso } & \multicolumn{2}{|c|}{$\begin{array}{c}\text { bloque de } \\
\text { deslizamiento } \\
\text { compacto (CSB) }\end{array}$} & \multicolumn{2}{|c|}{$\begin{array}{c}\text { Bloque de } \\
\text { deslizamiento con } \\
\text { sedimentos }\end{array}$} & \multicolumn{2}{|c|}{$\begin{array}{c}\text { Punta de } \\
\text { compresión de } \\
\text { deslizamientos } \\
\text { (SCT) }\end{array}$} \\
\hline & Ángulo ${ }^{\circ}$ & Altan & Ángulo ${ }^{\circ}$ & Altan & Ángulo ${ }^{\circ}$ & Altan & Ángulo ${ }^{\circ}$ & Altan \\
\hline Comau & $3.1-3.4$ & $17.3-17.7$ & $0.1-3.4$ & $4.5-17.7$ & $1.7-3.0$ & $14.7-17.2$ & 0.5 & 9.0 \\
\hline Puyuguapi & 1.3 & 13.6 & $0.4-0.7$ & $8.4-11.1$ & $0.3-1.7$ & $6.4-14.8$ & 0.1 & 3.2 \\
\hline Cupquelán & 7.6 & 21.3 & 1.8 & 15.0 & - & - & 0.4 & 8.4 \\
\hline Elefantes & 1.2 & 13.0 & - & - & .- & - & 0.4 & 8.4 \\
\hline
\end{tabular}


Tabla 3. Pendientes de vertientes II: Fiordos con explanadas de represamiento y acumulaciones caóticas. Table 3. Slope angles: Fjords with ponding esplanades and chaotic accumulations. Symbols as in figures 2 - 9.

\begin{tabular}{|c|c|c|c|c|c|c|}
\hline \multirow[t]{2}{*}{ Fiordo } & \multicolumn{2}{|c|}{ Umbral rocoso } & \multicolumn{2}{|c|}{$\begin{array}{l}\text { Acumulación caótica } \\
\text { de umbral (A) }\end{array}$} & \multicolumn{2}{|c|}{$\begin{array}{l}\text { Acumulación caótica } \\
\text { de explanada de } \\
\text { represamiento }(B)\end{array}$} \\
\hline & Ángulo ${ }^{\circ}$ & Altan & Ángulo & Altan & Ángulo & Altan \\
\hline Jacaf $4425-7250$ & 13.1 & 23.7 & 42.0 & 29.5 & $12.4-23.7$ & 23.4-26.4 \\
\hline Id. $4421-7256$ & $9.6-30.5$ & $22.3-27.7$ & $14.0-18.3$ & $24.0-25.2$ & $18.3-31.0-40.0$ & $25.2-27.8-29.2$ \\
\hline Id. 4418-7304 & $21.8-45.0-51.6$ & $26.1-30.0-31.0$ & $31.0-42.0-72.0$ & $27.8-29.5-34.9$ & $22.0-31.0-54.5$ & $26.1-27.8-31.5$ \\
\hline Id. 4414-7317 & $13.0-24.0$ & $23.6-26.5$ & $11.3-13.0$ & $23.0-23.6$ & $6.0-36.0$ & $20.2-28.6$ \\
\hline
\end{tabular}

\section{Cuencas de fiordo con explanada de represamiento uniforme}

En las Figs. 2 a 5 se aprecia la estructuración de los fondos planos por capas laminadas de sedimentos. Estas estructuras son llamadas "ponded" por autores anglosajones. Por esto, se propone denominar explanada de represamiento al llano de fondo de cuenca. Su pendiente no aparece en las tablas por tratarse de formas horizontales. En las partes distales con respecto a las vertientes, el espesor y aspecto de los reflectores fuertes y medios no es constante. Su límite con las unidades transparentes puede ser brusco o irregular, correspondiéndose en general con el modelo de estratificación gradual. La diferencia con éste consiste en que el límite irregular puede estar en cualquiera de los dos bordes del reflector fuerte o incluso en ambos, por lo que una disposición grano-decrecente en las capas no puede deducirse siempre. Las muestras de "box corer" o "gravity corer" son superficiales y decimétricas: no agregan información para esto. Aún así, las propiedades de penetración del sonar a $3.5 \mathrm{k} \mathrm{Hz}$ permiten suponer que las capas están compuestas de sedimentos finos. La comparación de cubetas contiguas en la cuenca del fiordo Comau (Fig. 2) refuerza esta idea. Sólo donde hay multicapas las ondas penetran en material no transparente. Además, la potencia total de los cortes muestra dos secciones de espesor variable: la superior, con capas laminadas prácticamente intactas ( 5 a $14 \mathrm{~m}$ de potencia) y la inferior, con capas comúnmente disturbadas (potencia desconocida). Entre ambas, la discordancia se ve hacia los bordes de la cubeta, indicando por lo menos dos etapas de la estructuración multilaminar, con deformación de la inferior.
Para explicar esto último, es necesario describir los fenómenos de borde de cubeta en vertiente de umbral (Figs. 2 a 5 y Tabla 2). El bloque de deslizamiento compacto existe en vertientes suaves y se caracteriza por la mantención de la estructura laminada de los sedimentos sobre vertientes de umbral rocoso también suaves. Su carácter deslizante se deduce de la deformación de los sedimentos vertiente abajo. El bloque de deslizamiento con sedimentos remoldeados se presenta hacia abajo del compacto, como resultado del efecto de éste en la compresión de las capas laminadas, remoldeándolas. La punta de compresión de deslizamiento tiene una superficie rugosa compuesta de sedimentos deformados, pero una pendiente general muy suave; es la expresión de deformación distal y está en contacto directo con el cuerpo de la explanada de represamiento. Para las tres formas nombradas hay ejemplares superficiales (superiores) y fosilizados (inferiores), relacionándose con las dos partes reconocidas en la estructura de la explanada. Si la punta de compresión es superficial, la superficie de la explanada próxima a ella está abombada, rasgo que se propone llamar abombamiento proximal; si está fosilizada, su contacto es directo con el cuerpo subterráneo deformado de la explanada.

Se demuestra entonces que la estructuración de la explanada de fondo de depresión está en relación con la dinámica de las vertientes de los bordes, por lo menos en dos grandes fases de la evolución.

\section{Cuencas de fiordo con explanadas de represamiento y acumulaciones caóticas}

El único caso de esta categoría es el Canal Jacaf. 


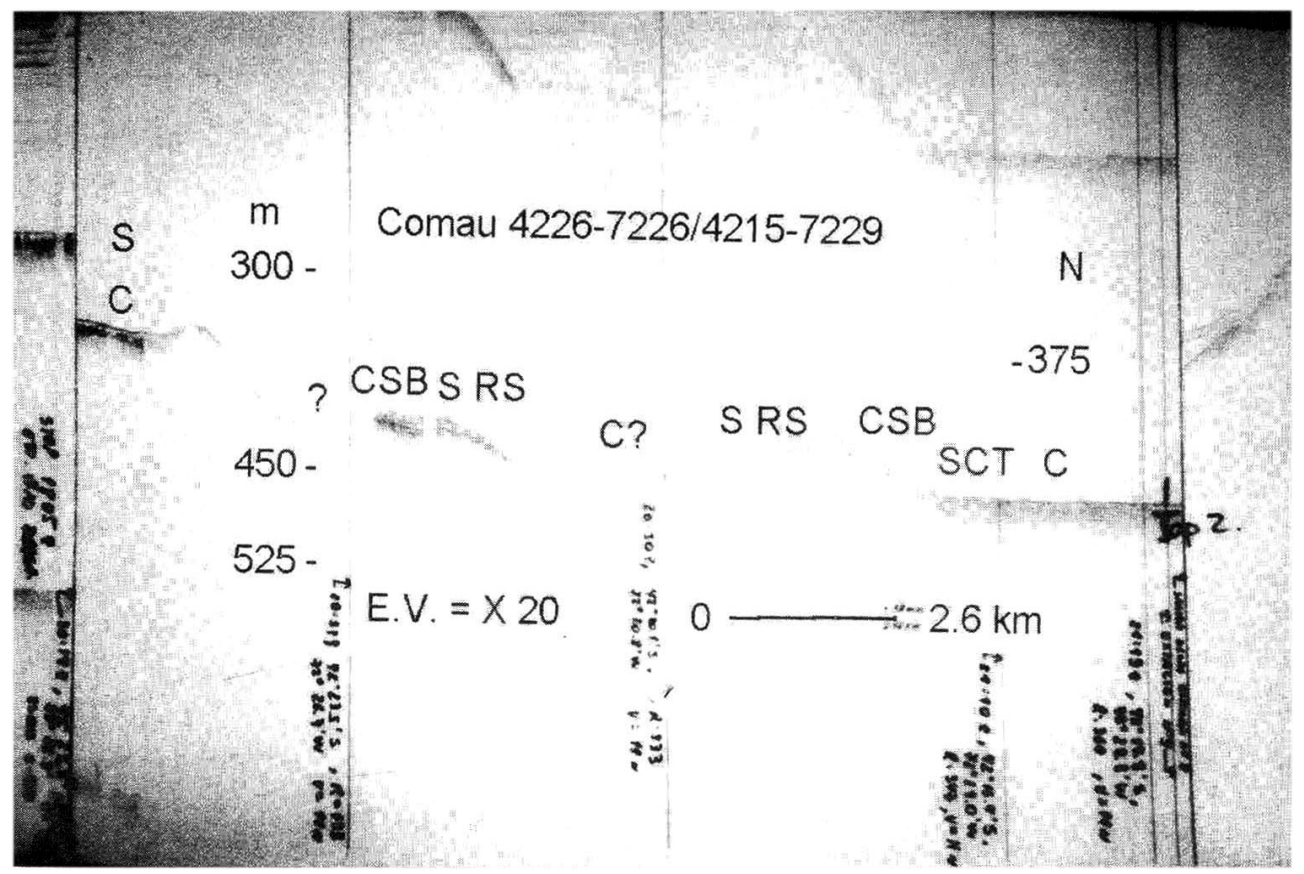

Fig. 2. Fiordo Comau: registro de ecosonda a $3.5 \mathrm{k} \mathrm{Hz}$; CSB= Bloque de deslizamiento compacto; $\mathrm{S} R S=\mathrm{Desliza}-$ miento con sedimentos remoldeados; $\mathrm{SCT}=$ Punta de compresión de deslizamiento; $\mathrm{C}=$ Explanada de represamiento. Nótese la diferencia de comportamiento acústico entre $\mathbf{C}$ y $\mathbf{C}$ ?, atribuida a diferencias granulométricas.

Fig. 2. Comau fjord: $3.5 \mathrm{k}$ Hz echo-sounder record; $\mathrm{CSB}=$ Compact slide block; $S R S=$ Slide remolded sediments; $\mathrm{SCT}=$ Slide compression toe; $\mathrm{C}=$ Ponding esplanade. Note the difference of acoustic feature between $\mathrm{C}$ and $\mathrm{C}$ ?, which is thought to represent granulometric differences.

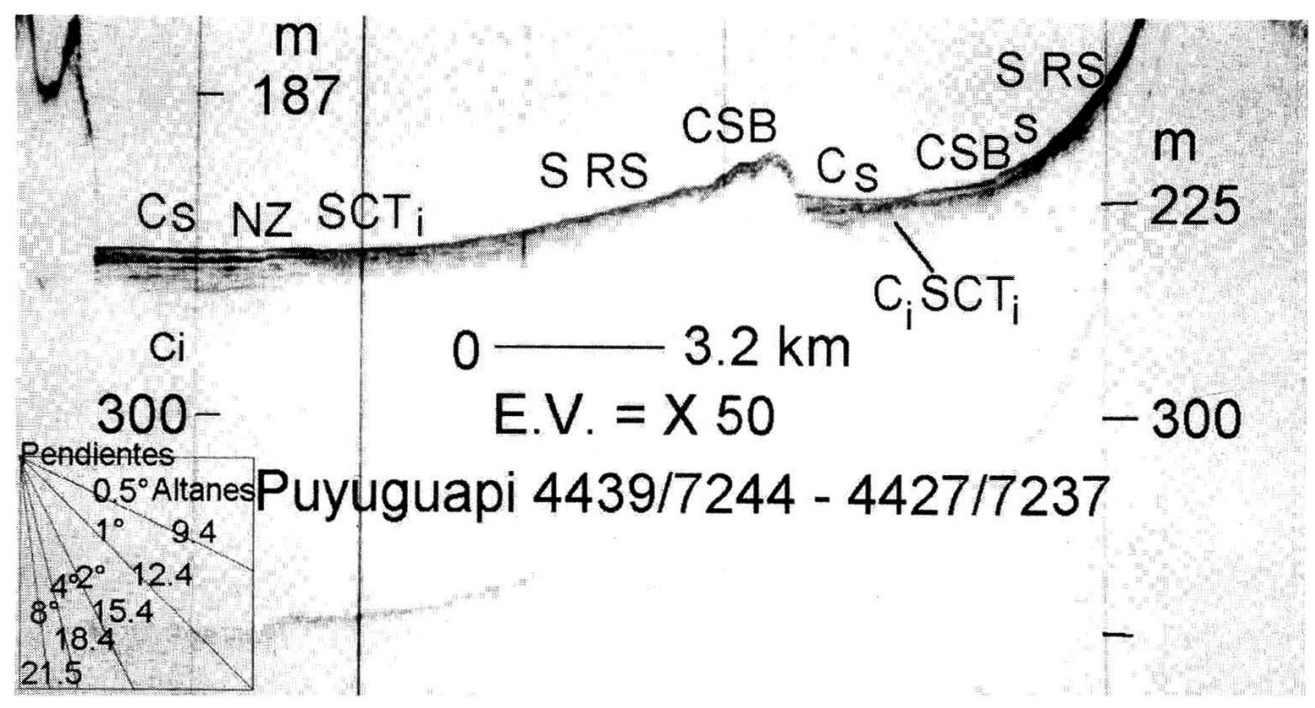

Fig. 3. Fiordo Puyuguapi: registro acústico a $3.5 \mathrm{k} \mathrm{Hz}$, cubeta interna; CSB= Bloque de deslizamiento compacto; $\mathrm{S}$ RS= Deslizamiento con sedimentos remoldeados; $\mathrm{SCT}=$ Punta de compresión de deslizamiento; $\mathrm{C}=$ explanada de represamiento; $N Z=$ Zona nodal de inflexión entre vertiente y explanada. Obsérvese la fosilización de las formas deprimidas de la fase inferior por las láminas de la fase superior.

Fig. 3. Puyuguapi fjord: $3.5 \mathrm{k} \mathrm{Hz}$ acoustic record, inner through; $\mathrm{CSB}=\mathrm{Compact}$ slide block; $\mathrm{SRS}=\mathrm{Slide}$ remolded sediment; $\mathrm{SCT}=$ Slide compression toe; $\mathrm{C}=$ ponding esplanade; $\mathbf{N Z}=$ nodal zone of inflection between slope and esplanade. Observe in what a manner the structures of the upper phase are overlying those of the lower one. 


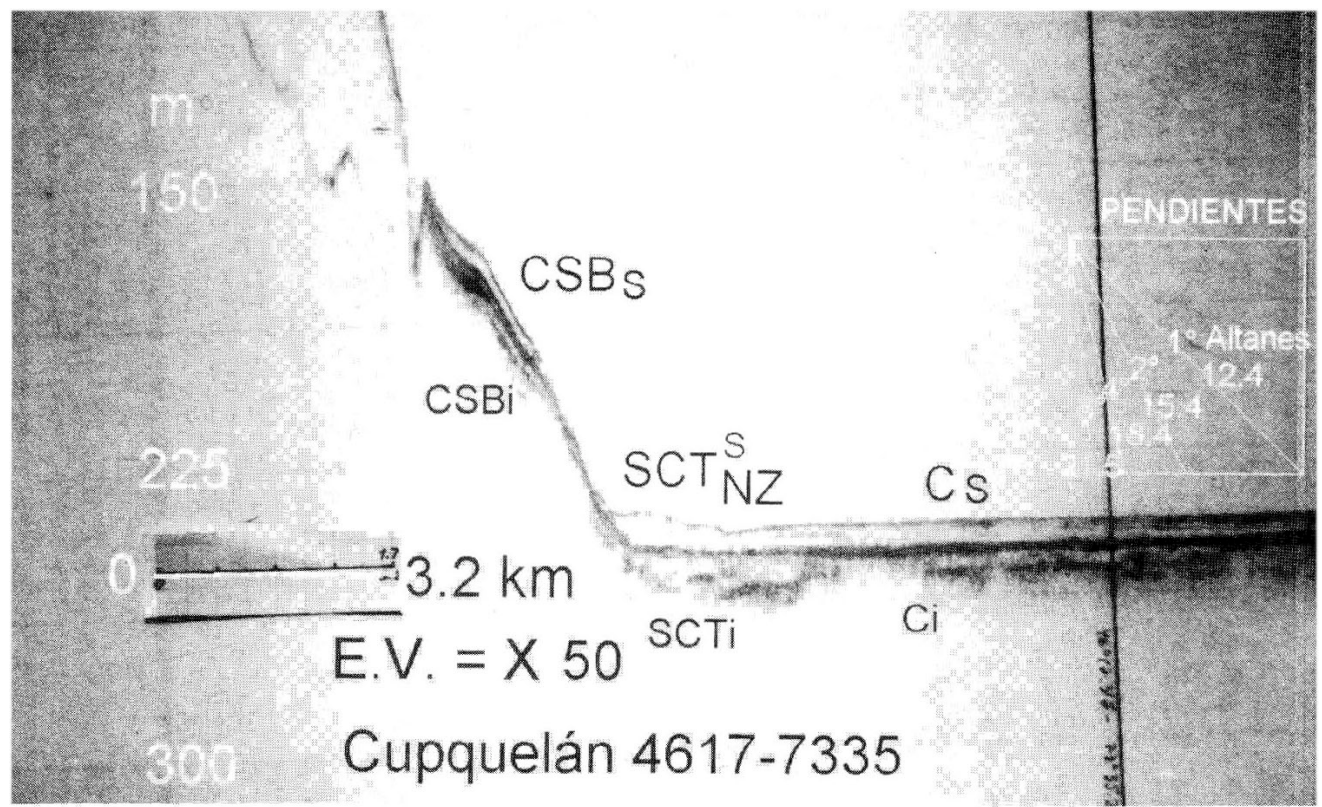

Fig. 4. Fiordo Cupquelán: contacto entre cuenca y umbral externo; CSB = Bloque de deslizamiento compacto; SCT = Punta de compresión de deslizamiento; NZ = Zona nodal de contacto entre dominio de deslizamientos y explanada C. Adviértase la convexidad muy suave entre NZ y C: se trata del abombamiento proximal. El fondo irregular contiene los sedimentos de la fase inferior, subyaciendo a las capas de la fase superior.

Fig. 4. Cupquelán fjord: echo-sounder record, contact between outer basin and threshold; CSB = Compact slide block; SCT = Slide compression toe; $\mathrm{NZ}=$ Nodal zone of contact between slide dominion and esplanade $\mathrm{C}$. Perceive the very gentle convexity between $\mathrm{NZ}$ and $\mathrm{C}$ : it is the proximal bending. In the irregular bottom, the sediments of the lower phase are underlying the beds of the upper phase.

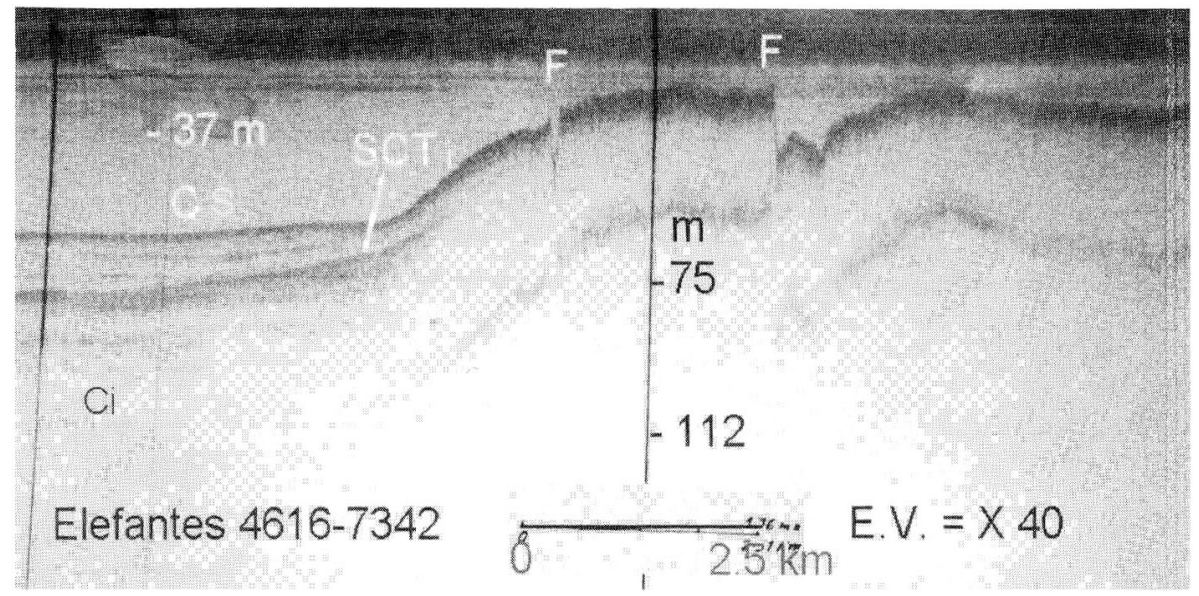

Fig. 5. Fiordo Elefantes: registro de ecosonda, cubeta interna; F= Fractura; $\mathrm{SCT}=$ Punta de compresión de deslizamiento; $\mathbf{C}=$ Explanada de represamiento. Atiéndase a la discordancia entre las capas de las fases inferior y superior y al carácter fósil de la punta de compresión de deslizamiento. Entre la base de vertiente y $\mathrm{C}$ se extiende el abombamiento proximal.

Fig. 5. Elefantes fjord: echo-sounder record, inner through; $\mathrm{F}=$ Fracture; $\mathrm{SCT}=\mathrm{Slide}$ compression toe; $\mathrm{C}=$ Ponding esplanade. See the discordance between the upper and lower phases and the fossil character of SCT. Between the slope base and $\mathrm{C}$ appears the proximal bending. 
(Figs. 6, 7 y 8; Tabla 3), cuya cuenca es la más compleja, con cubetas más profundas y umbrales más escarpados. La explanada de represamiento está adaptada aquí a cubetas estrechas y los reflectores fuertes que representan a las capas laminadas son más nítidos y paralelos que sus homólogos de la categoría anterior. Además, sus límites superior e inferior con las unidades transparentes son más homogéneamente bruscos; las irregularidades o sectores gradados son excepcionales. Cada límite debe representar cambios drásticos entre las tasas de sedimentación de unidades transparentes y reflectoras.

Las acumulaciones caóticas son las formas de mayor pendiente y están representadas por reflectores internos incoherentes y tenues correspondientes a mantos superpuestos de espesor variable, internamente transparentes y que revelan distintos momentos de depositación. Su aspecto, visto al $33 \%$ de la distorsión original de la escala vertical, es de lomas con vertientes multisecuenciuales, cuyas convexidades están condicionadas por la superposición de mantos de sedimentos. Se puede reconocer dos categorías básicas: de umbral y de explanada de represamiento. La primera existe en la cima o en la vertiente de un umbral rocoso; su altura relativa, variable, puede sobrepasar los $300 \mathrm{~m}$, siendo la categoría de mayor pendiente. La segunda se emplaza en el interior de la explanada de represamiento; su base está siempre fosilizada por las capas de estructura laminar, cuyo espesor puede ser de más de $70 \mathrm{~m}$; desde allí, su altura relativa puede sobrepasar los $150 \mathrm{~m}$; para la parte que emerge de las capas laminadas, su pendiente es algo menor que en las formas de umbral; algunos ejemplares están semicubiertos por las capas laminadas superficiales, las cuales están abombadas por presencia cercana de la cima semi-subterránea.

Un elemento importante aparece en la zona de contacto entre la acumulación caótica y las capas laminadas de la explanada (D en Figs. 6, 7 y 8). La vertiente de aquella engrana con éstas formando cuñas, que se ven en el corte en forma de dientes de serrucho. Por razones dadas en la discusión, estas cuñas serán denominadas provisoriamente lenguas de material caótico. Cada lengua sobreyace a un reflector fuerte y sólo muestra cambio lateral de facies con la lámina acústicamente transparente, fundiéndose frecuentemente con ella. O sea, cuando se formó la lengua, la superficie de la explanada estaba compuesta por el material del reflector, sedimentado al final de una secuencia, después del material transparente. Sobre el reflector y sincrónicamente con la lengua, debe haberse formado la lámina transparente de la nueva secuencia y así en seguida (Fig. 9).

Consecuentemente, estos hechos indican que las formas de acumulación caótica y las estructuras laminadas de la explanada deben haberse formado paralela y sincrónicamente.

\section{DISCUSIÓN}

En las cuencas con explanada de represamiento uniforme, es manifiesta la conexión genética de las capas del fondo con los materiales de las vertientes de umbral, como ya había aparecido en algunas otras experiencias (notablemente en The Grape Team, 1990). Los movimientos en masa reconocidos en las vertientes tienen patrones coincidentes con los observados por análisis o experimentos anteriores (Porebski et al., 1991; Barnes y Lewis, 1991; Myrow y Hiscott, 1991; Trincardi et al., 1995) y en una discusión taxonómica reciente (Mulder y Cochonat, 1996). El seguimiento de la cadena genética en la región estudiada debe hacerse considerando que las pendientes de vertiente de umbral son bajas (difícilmente pueden activar movimientos en masa rápidos) y que los materiales están estratificados en forma parecida a la de las capas laminadas. La frecuencia con que aparecen deslizamientos compactos es congruente con la suavidad de las pendientes. Las formas de compresión vertiente abajo (sedimentos remoldeados y punta de compresión) deben haberse producido también lentamente, sin perjuicio de incidencias cataclismáticas sísmicas, como puede deducirse de otros casos (Mosher et al., 1994; Anastasakis y Piper, 1991) y de rugosidades aisladas en estructuras laminadas de estos ejemplos. Por otra parte, si las capas de la explanada tienen detalles de estratificación cambiante y sólo algunas coinciden con la posición de la punta de compresión de deslizamiento, lo más probable es que ellas se relacionen con procesos diferentes en las vertientes, desde licuefacciones hasta deslizamientos, activando el flujo licuefacto, la corriente de turbidez y el flujo de deslizamiento, como deducen Myrow y Hiscott (1991) a partir de observaciones subaéreas. Ello debiera tenerse en cuenta en las discusiones sobre turbiditas (como Rothman et al., 1994 y Hiscott, 1994). En otras palabras, el proceso de corrientes de turbidez parece no explicar exclusivamente estas morfoestructuras.

Estas características pueden aplicarse a las dos 


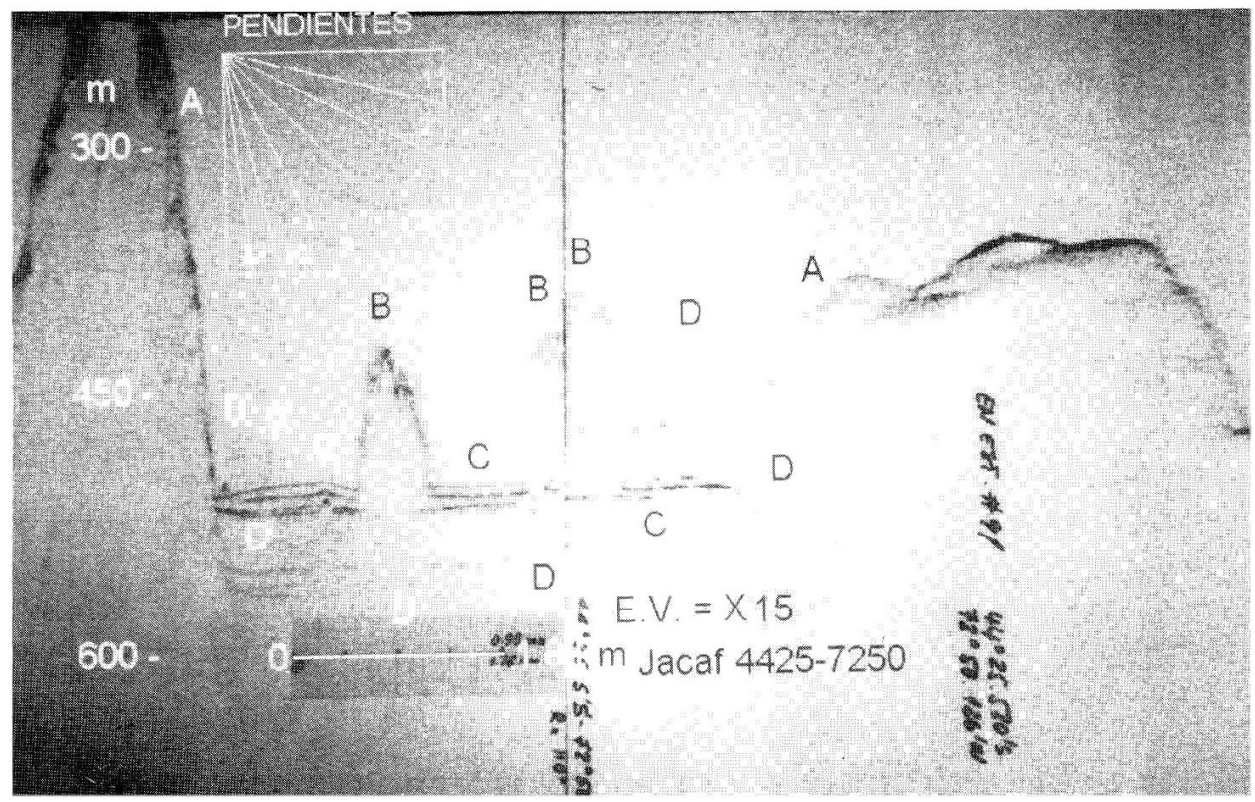

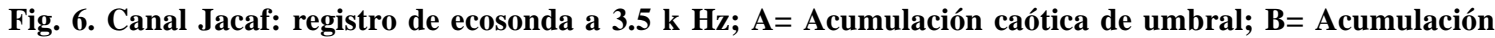
caótica de explanada de represamiento $C ; D=$ Lenguas de material caótico en engranamiento de $A$ o $B$ con las capas de $C$. Adviértase casos de $B$ casi enterrados por capas de $C$.

Fig. 6. Canal Jacaf: $3.5 \mathrm{k}$ Hz echo-sounder record; $A=$ Threshold chaotic accumulation; $B=$ Chaotic accumulation of ponding esplanade $C ; D=$ Tongues of chaotic material resulting of the interbedding between $A$ or $B$ and beds of $C$. Observe cases of $B$ almost buried by beds of $C$.
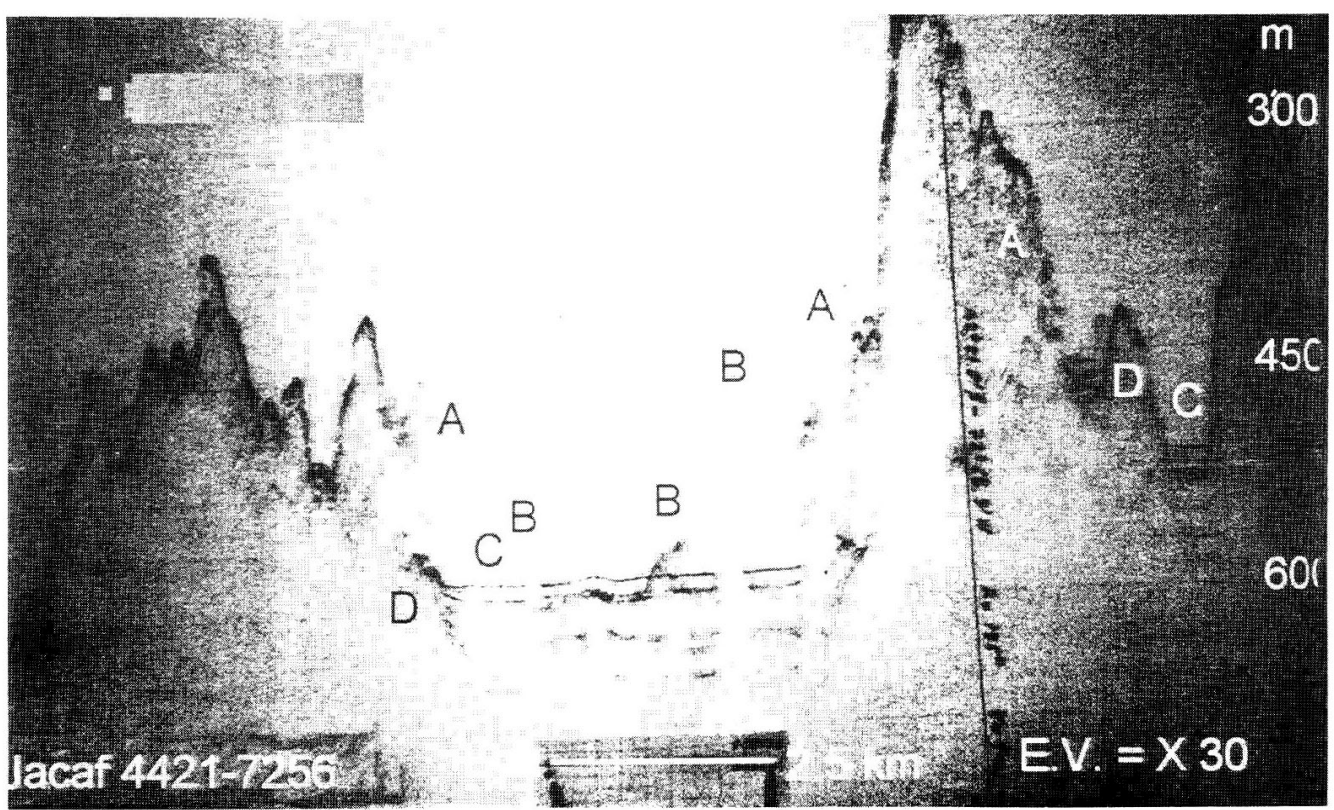

Fig. 7. Canal Jacaf: aplicar descripción de Fig. 6.

Fig. 7. Canal Jacaf: the same symbols of Fig. 6. 


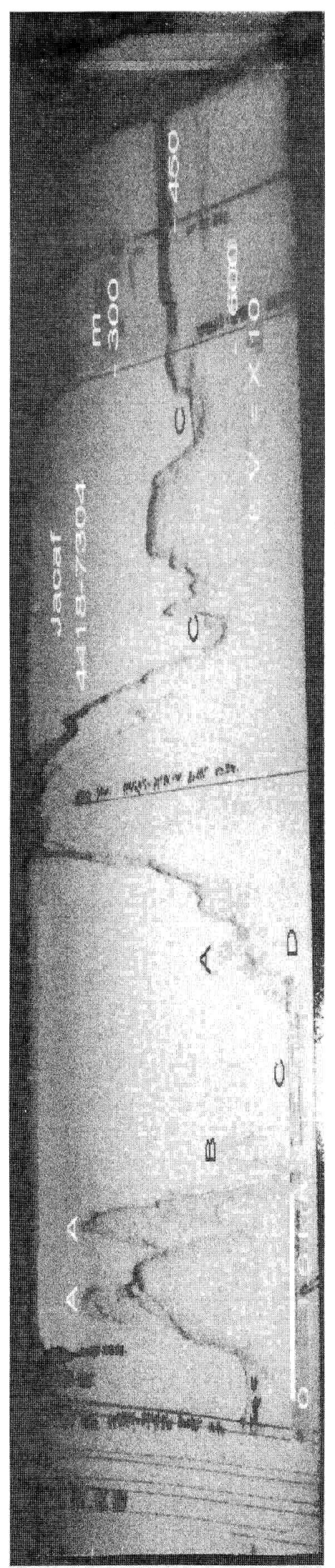

fases principales en la evolución de los fondos de cuenca, por no haber evidencia de otros tipos de estratificación en las vertientes. Para la fase inferior, hay que ver la probabilidad de su relación con la Última Glaciación documentada por Heusser (1990); si fuera contemporánea a ésta, podría ser que la laminación de sedimentos en vertientes y fondos de cuencas se debiera a la existencia de lagos subglaciales, parecidos a los descubiertos en la Antártica por Siegert et al. (1996). Es probable que esto explique las capas más profundas, quizás no documentadas en los registros, y más antiguas que la fase inferior. Para ésta, una asociación con la operación glacial es especulativa. Más claro parece el caso de la fase superior, por su encajamiento en la inferior, el estado más intacto de sus formas y la datación de sus capas superficiales . La datación por Pb210 (Salamanca, 1996) se puede aplicar a la superficie de esta fase; da, como edades mayores, 120 años para $23 \mathrm{~cm}$ y 106 años para $50 \mathrm{~cm}$ superficiales. Asumiendo -con cierto margen de especulaciónuna tasa de sedimentación más o menos constante en la fase superior, se puede pensar en edades entre 2600 y 7300 años en el primer caso y entre 1000 y 3000 años en el segundo, para potencias entre 5 y $14 \mathrm{~m}$ respectivamente.

En las cuencas con explanada de represamiento y acumulaciones caóticas de Canal Jacaf, la presencia de estas últimas y el conocimiento del campo de la Última Glaciación permiten aplicar la teoría sobre procesos glaciomarinos de borde de calota, reforzada con observaciones sobre mecanismos de fluctuación del frente de hielo (Frezzotti, 1997), asociados con quebraduras y procesos de desprendimiento o calving (Vaughan, 1993; Harry et al., 1990), y sobre la existencia de lagos subglaciales (Siegert et al., 1996). Contrastando los modelos de sedimentación en Fiordo Ferrar, Antártica (Barrett y Hambrey, 1992), los casos de Canal Jacaf pueden interpretarse como generados en zonas proximales al límite entre hielo varado en umbrales y hielo flotante, pero bajo este último. Se puede asumir que los caóticos son diamicton masivo y las capas laminadas, diamicton estratificado, correspondiendo genéticamente a till de amontonamiento ("lodgement till") y till acuayacente ("waterlain till") respectivamente. Desde el punto de vista morfogenético, tanto las dos últimas formas como las lenguas de material caótico que las engranan, pueden ser explicadas usando el modelo de la "till tongue" o lengua de till (King y Fader, 1986, en Josenhans y Fader, 1989) y las observaciones sobre "lift off" moraines o 


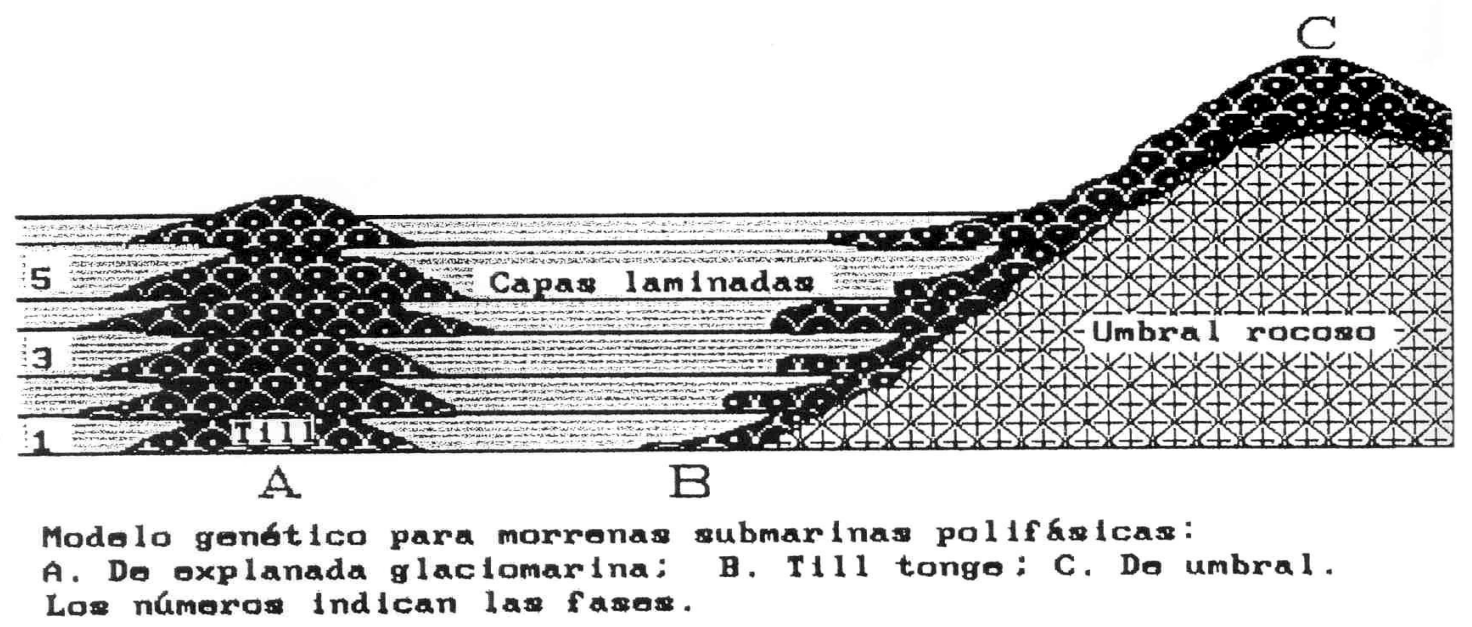

Fig. 9. Descripción en la figura y en el texto. Comparar con Figs. 6, 7 y 8.

Fig. 9. Description in the figure and in the text. Compare with Figs. 6, 7 and 8.

morrenas de despegue (Josenhans y Fader, 1989). Estas últimas son interpretadas como producto de sedimentación en fracturas en la base de una placa de hielo durante su despegue inicial desde el fondo; su engranamiento con las capas de la explanada de represamiento sugiere poco movimiento horizontal del hielo. En todo caso, no es seguro que el mecanismo constructivo de las morrenas haya sido por "lift off". Conjuntamente, el modelo de desarrollo de lenguas de till, atribuye su formación a una escalonada elevación de la línea de flotación del hielo a medida que se acumula el till de amontonamiento. En el caso analizado, el engranamiento debiera ser consubstancial con la siguiente distribución granulométrica: diamicton masivo para la lengua de till; parte gruesa del till para la capa laminada acústicamente transparente, y parte fina del till con cohesión alta para la capa reflector. Esto significa que la turbidez generada por cada diseminación lateral de till es la responsable de la constitución de la capa reflectora, por depositación tardía.

\section{CONCLUSIONES}

El problema genético de los fondos de fiordos analizados puede formularse como sigue:

Explicación de la existencia de dos fases morfogenéticas en explanadas de represamiento uniformes.
Determinación del mecanismo de instalación de la fase inferior.

Explicación del tipo de emplazamiento de las explanadas de represamiento que alternan con formas de diamicton masivo.

Probabilidad de que las explanadas de represamiento uniformes también se hayan originado a partir de formas de diamicton masivo, lo que hace necesario un seguimiento en distintos fiordos del resto de Patagonia.

Si se demuestra que en la respuesta el punto 4 es positiva, el diamicton masivo debiera considerarse como el primer eslabón en la cadena genética de los fondos de fiordo.

Debido a la importancia del punto anterior, se propone el nombre de morrenas polifásicas para las formas que resulten de la interacción entre las varias fases de acumulación de diamicton masivo y la formación de capas laminadas de explanada. El modelo genético de la Fig. 9 indica la formación de tres categorías de morrenas polifásicas: de explanada glaciomarina, "till tongue" y de umbral.

\section{AGRADECIMIENTOS}

El autor expresa su reconocimiento al Comité Oceanográfico Nacional, a su Secretario Ejecutivo Sr. Mario Cáceres, al personal de AGOR Vidal 
Gormaz, al Sr. Cristian Rodrigo y al Prof. Eduardo Valenzuela, por haber puesto a su disposición el material analizado.

\section{REFERENCIAS BIBLIOGRÁFICAS}

Anastasakis, G.C. y D.J.W. Piper. 1991. The character of seismo-turbidites in the S-1 sapropel, Zakinthos and Strofadhes basins, Greece. Sedimentology, 38: 717-733.

Araya-Vergara, J.F. 1996. Geomorfología comparada de los fiordos de Chiloé y Aysén. In: Comité Oceanográfico Nacional-Chile (ed.) Resultados crucero Cimar-Fiordo 1: Resúmenes ampliados. CONA, Valparaíso, pp. 15-17.

Barnes, Ph.M y K.B. Lewis. 1991. Sheet slides and rotational failures on a convergent margin: the Kidnappers Slide, New Zealand. Sedimentology, 38: 205-221.

Barrett, P.J. y M.J. Hambrey. 1992. Plio-Pleistocene sedimentation in Ferrar Fiord, Antarctica. Sedimentology, 39: 109-123.

Carlson, P.R. 1989. Seismic reflection characteristics of glacial and glaciomarine sediment in the Gulf of Alaska and adjacent fjords. Marine Geol., 85: 391-416.

Frezzotti, M. 1997. Ice front fluctuation, iceberg calving flux and mass balance of Victoria Land glaciers. Antarctic Sci., 9(1): 61-73.

Hansom, J.D. y C.P. Flint. 1989. Holocene ice fluctuations on Brabant Island, Antarctic Peninsula. Antarctic Sci., 1(2): 165-166.

Harry, J.R., S.S. Jacobs y D. Barnett. 1990. The calving and drift of iceberg B-9 in the Ross Sea, Antarctica. Antarctic Sci., 2(3): 243-257.

Heusser, C.J. 1990. Chilotan piedmont glacier in the southern Andes during the last glacial maximum. R. Geol. de Chile, 17: 3-18.

Heusser, C.J. y R.F. Flint. 1977. Quaternary glaciations and environments of northern Isla Chiloé, Chile. Geology, 5: 305-308.

Recibido el 4 de julio de 1997. Aceptado el 27 de mayo de 1998.
Hiscott, R.N. 1994. Loss of capacity, not competence, as the fundamental process goberning deposition from turbidity currents. J. os Sedim. Res., A64(2): 209-214.

Josenhans, H.W. y G.B.J. Fader. 1989. A comparison of models of glacial sedimentation along the eastern Canadian margin. Marine Geol., 85: 273-300.

Mosher, D.C., K. Moran y R.N. Hiscott. 1994. Late Quaternary sediment, sediment mass flow processes and slope stability on the Scotian Slope, Canada. Sedimentology, 41: 1039-1061.

Mulder, Th. y P. Cochonat. 1996. Classification of offshore mass movements. J. of Sedim. Res., 66(1): 43-57.

Myrow, P.M. y R.N. Hiscott. 1991. Shallow-water gravity-flow deposits, Chapel Island Formation, Southern Newfounland, Canada. Sedimentology, 38: 935-959.

Porebski, S.J., D. Meischner y K. Görlich. 1991. Quaternary mud turbidites from the South Shetland Trench (West Antarctica): recognition and implications for turbidite facies modeling. Sedimentology, 38: 691-715.

Rothman, D.H., J.P. Grotzinger y P. Flemings. 1994. Scaling in turbidite deposition. J. of Sedim. Res., A64(1): 59-67.

Salamanca, M.A. 1996. Geocronología de sedimentos marinos de la zona de fiordos de la XI Región. In: Comité Oceanográfico Nacional-Chile (ed.) Resultados Crucero Cimar-Fiordo 1: Resúmenes ampliados. CONA, Valparaíso, pp. 64-68.

Siegert, M.J., J.A. Dowdeswell, M.R. Gorman y N.F. Mcintyre. 1996. An inventory of sub-glacial Antarctic lakes. Antarctic Sci., 8(3): 281-286.

The Grape Team. 1990. Preliminary results of seismic reflection investigations and associated geophysical studie in the area of the Antarctic Peninsula. Antarctic Sci., 2(3): 223-234.

Trincardi, F., A. Correggiari, M.E. Field y W.R. Normark. 1995. Turbidite deposition from multiple sources: Quaternary Paola Basin (Eastern Tyrrenian Sea). J. of Sedim. Res., B65 (4): 469483.

Vaughan, D.G. 1993. Implications of the break-up of Wordie Ice Shelf, Antarctica for sea level. Antarctic Sci., 5(4): 403-408. 